\title{
Dalla diagnosi di Insufficienza Renale all'Emodialisi, una vita che cambia
}

\author{
Barbara Pittau \\ UOC Nefrologia e Dialisi, PO San Francesco, ATS Sardegna, ASSL Nuoro, Nuoro
}

\begin{abstract}
From the diagnosis of renal failure to hemodialysis: a life change
The goal of this project was to explore the experiences of patients and nurses in the care relationship and to highlight these experiences and feelings in their pathway. Listening carefully and giving importance to the story of patients and health professionals makes them appreciate their importance and uniqueness. Shared narration acquires a deep meaning because it conveys to the listener the values that determined the choices and actions of the storyteller. We gathered for the present study several narratives of patients; four of them have been selected here. For the narrative analysis we used the Qualitative Content Analysis approach, which aims to describe and highlight the characteristics of a phenomenon. From the analysis of the narrative texts four categories have been identified: hope/confidence, anxiety/fear, sense of impotence/disfavor, and empowerment/determination.
\end{abstract}

Keywords: Care relationship, Narration, Qualitative content analysis

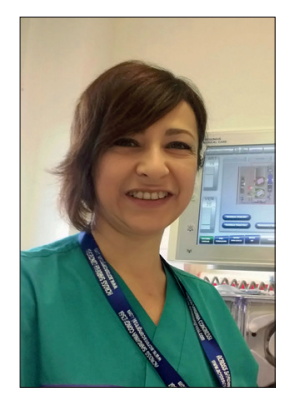

Barbara Pittau
"Spesso i pazienti soffrono di cose ben diverse da quelle indicate sulla loro cartella clinica. Se si pensasse a questo, molte loro sofferenze potrebbero essere alleviate"

Florence Nightingale

\section{Introduzione}

La possibilità di raccontarsi implica l'esistenza nel proprio animo di una sensibile dote umana, la capacità di ascoltare il prossimo, che nutre l'individuo. Mi chiamo Barbara e sono un'infermiera. Un anno fa mi si è offerta la possibilità di fare uno scambio compensativo con un collega dell'UOC di Nefrologia e Dialisi. Dopo qualche titubanza iniziale, la mia curiosità innata, la mia voglia di conoscere e di imparare cose nuove e la mia cronica

Accepted: April 12, 2017

Published online: July 19, 2017

Indirizzo per la corrispondenza:

Barbara Pittau

Via Biasi, 136

08100 Nuoro

barbara.pittau@live.it attitudine ad avere stimoli continui per poter andare avanti hanno avuto la meglio e ho accettato. Essendomi formata in Area Critica e avendo lavorato diversi anni in Rianimazione e Terapia Intensiva, l'emodialisi per me non era una cosa del tutto sconosciuta, ma lavorare con pazienti cronici è molto diverso. La scelta di approfondire il tema della narrazione in ambito sanitario nasce durante la partecipazione a un Laboratorio di Medicina Narrativa, in qualità di osservatrice e partecipante attiva, ed è stato un colpo di fulmine per me, che adoro ascoltare storie di vita e leggere biografie. È stata una bellissima esperienza incontrare queste persone che hanno messo a disposizione il loro vissuto da dializzati e/o trapiantati, da cui sono certamente uscita arricchita e preparata ad affrontare meglio il nuovo incarico. Questa esperienza è stata infatti per me un vero e proprio percorso di crescita, iniziato da un semplice interesse e diventato via via sempre più coinvolgente. Poter sperimentare realmente che cosa significhi diventare parte di un gruppo, condividendo emozioni, paure e gioie, arricchisce da un punto di vista umano e professionale. Da qui è nata una nuova passione, che va a integrare l'interesse che da tempo ho per la psicologia clinica, il counselling e la comunicazione.

La narrazione è uno strumento che ha la funzione di valorizzare e dare dignità alle persone e coesione all'interno dei team di lavoro. In questo modo emerge la specificità umana del lavoro al quale tutti partecipano e si dà loro consapevolezza. Dare spazio a pazienti e infermieri per narrare la propria storia significa mostrare sensibilità e rispetto altrui, condividere i pensieri, 
le emozioni, le responsabilità. Le narrazioni ci aiutano a vedere qualcosa che è davanti ai nostri occhi, spesso invisibile.

In chi partecipa ad ambiti in cui la narrazione è fondante, oltre alle principali conseguenze indicate da Charon (1), attenzione, rappresentazione e affiliazione, si può aggiungere l'effetto familiarità. Nella formazione narrativa delle professioni sanitarie i professionisti propongono una parte di loro, una parte della loro storia. Si espongono, si mettono in gioco, mettono a disposizione degli altri la loro esperienza in modo diretto. Costruiscono ponti di fiducia in direzione di chi li ascolta, condividono itinerari di crescita, di quotidianità, rivolgendosi agli astanti come se fossero persone conosciute da tempo. Attribuiscono intenzionalità comunicativa alla platea, pongono le basi per una reciprocità di rapporti. Fanno il primo passo verso una situazione di relazionalità basata sulla comune umanità. Si innesca appunto un processo di "familiarizzazione"

\section{Obiettivi}

Con questo proposito ho iniziato a lavorare a un progetto che prevede la raccolta di narrazioni di pazienti e di infermieri, con l'obiettivo di esplorare le loro esperienze nel rapporto di cura e per evidenziare i vissuti e le sensazioni provate all'interno del loro percorso.

\section{Materiali e metodi}

Per l'analisi delle narrazioni è stato utilizzato l'approccio della Qualitative Content Analysis, il cui obiettivo è quello di descrivere e far emergere un fenomeno e quindi raggiungere l'affidabilità, la credibilità e la trasferibilità dell'oggetto preso in esame. Si tratta di una tecnica di analisi dei dati che ha lo scopo di ridurre la grande varietà di informazioni qualitative, presenti in un documento verbale, in un insieme più piccolo e interpretabile di informazioni.

\section{Risultati}

Nell'analisi delle narrazioni dei pazienti la categoria emersa con più forza in tutte e quattro le interviste proposte è quella della speranza/fiducia (specialmente per un trapianto). Un'altra categoria emersa è quella del senso di impoten$\mathrm{za} / \mathrm{sconforto}$, soprattutto iniziale. Ritrovate le forze, dopo lo sconforto e il senso di impotenza, compare un'altra categoria individuata nelle analisi: quella dell'empowerment/determinazione (il ricevere, soprattutto dai familiari, la forza e il coraggio per affrontare una situazione difficile e quindi sentirsi determinati a combatterla).

Personalmente dai "miei" pazienti ho ricevuto molto di più: mi hanno fatto entrare nella loro vita, senza imbarazzo, mettendo a nudo sentimenti ed emozioni, che sulla carta non rendono. Ve li presento con una breve anamnesi e poi lascio la parola a loro.
L.F. Ventotto anni, in emodialisi da gennaio 2017. A 14 anni in seguito a una broncopolmonite, viene fatta diagnosi di miocardite dilatativa e sottoposto a trapianto di cuore d'urgenza. Portatore di pace-maker. Durante i controlli posttrapianto, tre anni fa viene fatta diagnosi di nefropatia.

1. Comunicazione della malattia. Reazioni, sentimenti, modalità

In maniera categorica la dottoressa mi ha fatto capire che ormai la dialisi era I'unico passo da compiere se non volevo morire. Avevo infatti sempre rimandato il momento finché mi sentivo bene. Ero accompagnato da un cugino che mi ha convinto a procedere. II medico mi ha proposto la dialisi peritoneale, ma ho scelto di fare l'emodialisi per una mia sicurezza.

2. Comunicazione della notizia alle persone vicine. Reazioni

Ho una figlia di 9 anni, l'ha presa con naturalezza. I miei genitori, con cui vivo, mi hanno detto che era ora che mi decidessi!

3. Supporto psicologico. Lo ritieni utile?

Nel mio caso no, I'ho sperimentato durante il trapianto di cuore, non ho sentito il bisogno di parlare con lo psicologo. Ma è una cosa molto personale. Lo ritengo invece indispensabile nel caso di bambini.

4. Disagio. Il tuo e quello degli altri. Come lo si affronta? Della malattia ne parlo normalmente. II disagio non è mio, ma degli altri. lo ho provato tanta confusione e fragilità al momento del ricovero in nefrologia. Paragonato al ricovero per il trapianto di cuore, per me è stato peggio, forse perché per il trapianto è successo tutto in fretta e non ero cosciente.

5. Lavoro. La malattia ha cambiato prospettiva nel lavoro $e$ in che modo?

Ho sempre lavorato, non mi sono mai fermato e se mi capita l'occasione lavoro.

6. Futuro. In che cosa stai investendo ora?

Su mia figlia.

7. Cosa ti cambierebbe la vita?

Vorrei poter dare un'occhiata a una vita parallela, senza la malattia, tipo sliding doors. Vorrei sapere come sarebbe andata.

8. Cosa hai imparato su te stesso da questa situazione? Che sono un combattente. E ho imparato a chiedere aiuto.

9. Un consiglio, un racconto, un suggerimento che daresti a qualcuno che sta per iniziare la dialisi o sta per affrontare il trapianto

Devi affrontarlo con forza interiore e, se possibile, con persone vicino.

G.B. Quarantanove anni. In emodialisi da 7 anni. Sottoposto due volte a trapianto di rene. Ha subito l'amputazione di una gamba per complicazioni seguite ai trapianti. 
1. Comunicazione della malattia. Reazioni, sentimenti, modalità

Ho una familiarità per il rene policistico. Mia nonna, mia mamma e mio fratello ne sono colpiti. Nonostante questo non ho fatto i controlli per tre anni. Durante una visita cardiologica hanno diagnosticato un'ipertensione, ma io ho tirato avanti un altro anno, finché i medici non sono stati categorici e mi hanno messo davanti alla realtà senza tanti giri di parole.

2. Comunicazione della notizia alle persone vicine. Reazioni

Ovviamente a casa lo sapevano, avevo aspettato anche troppo.

3. Supporto psicologico. Lo ritieni utile?

Sì, assolutamente. Soprattutto al momento della diagnosi e dell'inizio dialisi. Al Centro dove ho fatto i due trapianti c'era e per me era importante.

4. Disagio. II tuo e quello degli altri. Come lo si affronta? lo non provo disagio a dirlo. La gente reagisce o guardandoti come una bestia rara o con curiosità morbosa. Il disagio l'ho provato durante le visite per il trapianto, sono veramente invasive, alcune anche molto spiacevoli.

5. Lavoro. La malattia ha cambiato prospettiva nel lavoro $e$ in che modo?

Sono un geometra e sono senza lavoro. Inizialmente perché non potevo più recarmi sui siti a causa della gamba amputata, poi perché la crisi ha fatto diminuire molto il lavoro. Percepisco una pensione di invalidità e l'accompagnamento.

6. Futuro. In che cosa stai investendo ora?

Non ho una vita sentimentale, è difficile averne una dopo la malattia. Chi è sposato è perché lo ha fatto prima di ammalarsi. Ho la fortuna però di avere una famiglia che mi sta vicino e che mi dà la forza.

7. Cosa ti cambierebbe la vita?

Ho letto recentemente di un rene artificiale che si impianta e fa il lavoro della macchina per emodialisi. Per ora è in sperimentazione, ma quello sono sicuro che mi cambierebbe la vita.

8. Cosa hai imparato su te stesso da questa situazione?

Mi sono scoperto forte. Quando ho iniziato la dialisi ero terrorizzato dall'esperienza di mia madre. Stava sempre male, si collassava. Sono entrato in depressione e sono andato in analisi. Ma, come ho detto prima, la mia forza è data dalla mia famiglia, che sapeva cosa stavo affrontando.

9. Un consiglio, un racconto, un suggerimento che daresti a qualcuno che sta per iniziare la dialisi o sta per affrontare il trapianto

Sono stato trapiantato due volte di rene. Entrambe andate male. La prima per un errore medico. Era andato tutto bene. Togliendo i punti dei drenaggi strappano i punti della vescica e l'urina si sparge in addome. Durante l'intervento d'urgenza a cui sono stato sottoposto si forma uno pseudoaneurisma in arteria femorale sinistra, mi posizionano uno stent, ma si blocca la vascolarizzazione del rene trapiantato. Il rene è andato e ho iniziato una dialisi d'urgenza. A febbraio 2011 mi capita un'altra occasione di avere un rene. Dopo il trapianto sento un dolore come un forte crampo alla gamba sinistra, dove avevo avuto lo pseudoaneurisma. Lo stent si era ostruito, ma non è stato riconosciuto. A settembre trovano un trombo a livello della caviglia. Mi operano d'urgenza e mi amputano la gamba per l'insorgenza di un'infezione. Dovevo scegliere tra la gamba e il rene e ho perso entrambi. Ho provato la dialisi peritoneale. Un addestramento di 3 mesi e per i primi due anni è andata bene. Aumento di peso e le sacche di scambio da tre diventano cinque, per tre ore al giorno. Ero sempre ipoteso e mi collassavo. Vado avanti così per un anno, poi mi ricoverano e passo in emodialisi. Mi diagnosticano una cardiopatia, vengo operato nel 2015. Insorge un'endocardite batterica, mi sostituiscono le valvole e mi impiantano il pace-maker. Ho avuto poi una pielonefrite, avevo dei reni pieni di pus, uno pesava 4 chili, l'altro poco meno. Mi hanno impiantato anche un defibrillatore perché il mio cuore perdeva dei battiti e ora sembra vada meglio. Questa è la mia storia. Quello che posso dire a chi entra in dialisi è che è una cosa molto soggettiva. Consiglio di non seguire subito quello che dicono i medici, ma di trovare un proprio equilibrio con la conoscenza di se stessi.

C.C. Cinquanta anni. Nel 1992 la diagnosi di ipertensione arteriosa, con conseguente retinopatia e ipoacusia bilaterale. Successiva diagnosi di IRC da verosimile nefropatia da reflusso vescico-ureterale cronico. È stata in dialisi peritoneale e sottoposta a trapianto. A tutt'oggi è in emodialisi tri-settimanale. Ha partecipato al Laboratorio di Medicina Narrativa.

1. Comunicazione della malattia. Reazioni, sentimenti, modalità

Alla nascita ho avuto un'infezione urinaria non riconosciuta sino agli 11 mesi. Poi non mi sono stati fatti altri controlli per incuria medica, sino all'età di 22 anni, quando la malattia ormai era già cronica. Per 15 anni sono stata in cura e non ho dializzato. A 37 anni sono entrata in dialisi d'urgenza per i valori di creatinina altissimi, mi è crollato il mondo addosso. Per qualche mese ho rimandato, poi ho scelto la dialisi peritoneale, sia sotto consiglio medico e sia per la paura degli aghi. lo la preferisco per la metodica, per la libertà e la qualità di vita.

2. Comunicazione della notizia alle persone vicine. Reazioni

Mio marito non l'ha presa bene. La peritoneale la facevo tre volte al giorno e mi autogestivo, perché mio 
marito non l'accettava. Non è stato infatti il partner in addestramento. Anche alla telefonata per il trapianto lui diceva "Rifiuta", mio figlio che aveva solo 7 anni diceva "Vai!".

3. Supporto psicologico. Lo ritieni utile?

Personalmente non mi è servito, ho ricevuto l'aiuto che mi serviva dai nefrologi che sono stati chiari ed esaustivi e dalla mia famiglia. Ma se uno non ha questa fortuna, allora un aiuto psicologico è indispensabile. Prima di tutto infatti devi accettare tu questa nuova condizione di vita, anche perché non hai alternative, e poi la deve accettare chi sta intorno a te. Devo dire anche che avendo iniziato con la dialisi peritoneale a domicilio, non ho avuto un grosso trauma. Se avessi iniziato con l'emodialisi che sto facendo ora, non so se avrei reagito bene. Ti condiziona pesantemente la vita.

4. Disagio. Il tuo e quello degli altri. Come lo si affronta? Il disagio lo provo quando devo affrontare qualche visita, per il trapianto ne fai tante. Ma la peggiore resta la fistolografia: non sono stata informata adeguatamente su quello che avrei provato o potuto provare. Ho pianto per il forte dolore che non mi aspettavo e per non essere stata creduta. Ci vuole chiarezza nelle comunicazioni! I disagi li vivo in famiglia, non li affronto da sola, ma mia madre mi manca tantissimo. La sua morte mi ha paralizzata.

5. Lavoro. La malattia ha cambiato prospettiva nel lavoro e in che modo?

Prima della malattia ho lavorato poco, ma dopo il trapianto non ho più lavorato, troppa stanchezza, venivo ricoverata spesso. Ora ho l'inabilità e con l'adeguamento della pensione mi sono potuta permettere un aiuto in casa.

6. Futuro. In che cosa stai investendo ora?

Ho la fortuna di avere una bella famiglia. Ho un figlio, voluto a tutti i costi nonostante una gestosi e un parto precoce con taglio cesareo, che mi fa da stimolo. Vado avanti senza prospettive. La morte dei miei genitori (recentemente entrambi per tumore, N.d.A.) è stata troppo improvvisa e quindi vivo alla giornata, non faccio più progetti a lungo termine.

7. Cosa ti cambierebbe la vita?

Un altro trapianto.

8. Cosa hai imparato su te stessa da questa situazione? Che sono coraggiosa.

9. Un consiglio, un racconto, un suggerimento che daresti a qualcuno che sta per iniziare la dialisi o sta per affrontare il trapianto

Dopo solo 8 mesi dall'inizio della dialisi peritoneale mi chiamano per il trapianto, non volevo crederci! Sono partita con i miei genitori e mio marito. La famiglia mi aveva anche proposto il trapianto da vivente, mio fratello è compatibile, ma io non ho accettato. II trapianto è durato 10 anni, poi è andato male. A causa del farmaco antirigetto ho avuto una fibrosi polmonare. Ho ricominciato la peritoneale, poi è insorta una brutta infezione al peritoneo, il liquido è passato nei polmoni. Sono stata malissimo. Poi ho messo il catetere venoso centrale e di recente mi è stata confezionata la fistola e ho iniziato l'extracorporea. Nei giorni in cui la faccio, sto male. Nonostante questo penso sempre positivo, bisogna prendere la vita così come viene.

N.N. Quarantotto anni. In emodialisi da due anni, dopo una disfunzione renale.

1. Comunicazione della malattia. Reazioni, sentimenti, modalità

Comunicata dai medici in maniera chiara, ma gentile e sensibile.

2. Comunicazione della notizia alle persone vicine. Reazioni

Erano presenti mio fratello e mio padre. È stata una mazzata per tutti.

3. Supporto psicologico. Lo ritieni utile?

Un supporto psicologico sarebbe servito. Uno non sa nemmeno cosa sia la dialisi!

4. Disagio. Il tuo e quello degli altri. Come lo si affronta? Ne parlo tranquillamente. Le persone che non lo sanno quando mi incontrano, mi dicono che sono diverso, più magro (ero grasso, pesavo circa $60 \mathrm{~kg}$ in più) e quando spiego il perché sono molto imbarazzati. Tre giorni dopo il ricovero mi è stata confezionata una nefrostomia al rene sinistro per un anno e sette mesi. Ogni mese mi recavo in urologia per cambiare il catetere, poi ogni due mesi. Il personale era sempre gentile e competente. Mio fratello mi cambiava la sacca e se non c'era lui pagavo una ragazza per farlo. Poi si è formato un ematoma e quindi è stato necessario l'intervento di nefrectomia sinistra. Il disagio l'ho provato al ricovero, senza voglia di alimentarmi per l'umore nero che avevo. Inoltre la dieta da seguire è arrivata tardi, solo dopo tre mesi: sarebbe dovuta arrivare prima!

5. Lavoro. La malattia ha cambiato prospettiva nel lavoro e in che modo?

La malattia ha cambiato la vita lavorativa. Ho dovuto rinunciare al lavoro, facevo il cuoco nelle mense scolastiche. I soldi cambiano la vita... per esempio, se pagassero puntuali i rimborsi della benzina invece che con un ritardo di 6 mesi. Siamo ad aprile e ho ricevuto il rimborso di giugno-dicembre 2016. Pago 700-800 euro per essere accompagnato a fare la dialisi, percepisco 779 euro di pensione. Ricevo 100 euro a settimana dal comune, ma non copre le spese.

6. Futuro. In che cosa stai investendo ora? Ho imparato a vivere alla giornata, con filosofia.

7. Cosa ti cambierebbe la vita?

Un trapianto. Ora sono nella fase delle visite. 
8. Cosa hai imparato su te stesso da questa situazione? Che da solo non ce la fai. lo ho reagito con l'aiuto della famiglia. Sono tornato a vivere a casa di mia madre. Mi manca casa mia.

9. Un consiglio, un racconto, un suggerimento che daresti a qualcuno che sta per iniziare la dialisi o sta per affrontare il trapianto

Gli direi che ci vuole coraggio e tranquillità e di farsi aiutare da amici e parenti.

\section{Conclusioni}

Lo studio è stato condotto su un numero più ampio di narrazioni di pazienti, che volentieri si aprono a raccontare il loro vissuto. Purtroppo incontro invece molte difficoltà a raccogliere testimonianze da parte dei colleghi infermieri, motivo per cui il mio progetto per il momento è fermo.

\section{Ringraziamenti}

Ringrazio il Signor Gianpaolo Mele Corriga, scrittore, musicista e compositore, ricercatore etno-musicale, Maestro del folklore sardo e splendida persona, per aver dedicato del tempo a leggere il mio manoscritto e per i suoi preziosi suggerimenti durante le sedute dialitiche.

\section{Disclosures}

Financial support: No financial support was received for this submission. Conflict of interest: The author has no conflict of interest.

\section{Bibliografia}

1. Charon R. Narrative Medicine: Honoring the Stories of Illness. New York: Oxford University Press 2006. 